\title{
Origin of Giant Magnetoresistance in Granular Magnetic Systems
}

\author{
Minoru KUME, Atsushi MAEDA, Satoru OIKAWA, and Kazuhiko KUROKI \\ New Materials Research Center, SANYO Electric Co., Ltd., 1-1 Dainichi-higashimachi, Moriguchi, Osaka 570, Japan
}

\begin{abstract}
Granular Co-Ag films go through a steep demagnetization process when a permalloy layer is added to them, but slow saturation is maintained in the magnetoresistance (MR) curves, In addition, granular Fe-Ag films prepared under a magnetic field, in which strong magnetic anisotropy is induced, have MR characteristics that are isotropic. This disagreement between MR and magnetic characteristics indicates that ferromagnetic granules are not responsible for the giant MR (GMR) effect. The MR ratio of the granular Fe-Ag film considerably increases when the thickness of the film is less than $20 \mathrm{~nm}$. For such ultra-thin films, the MR curves and the magnetization curves with slow saturation and no hysteresis have features that correspond well with each other. These results show that superparamagnetism causes the GMR effect in granular systems.
\end{abstract}

\section{INTRODUCTION}

The giant magnetoresistance (GMR) effect in the $\mathrm{Fe} / \mathrm{Cr}$ multilayer [1] is a recent finding that has led to a great deal of investigations into the magnetotransport properties of various multilayered and sandwiched materials. This effect is believed to originate from interfacial spin-dependent scattering [2-5], since the multilayer materials have physical properties that are strongly influenced by interlayer diffusion. The GMR effect has also been found in some structures of granular materials comprising mutually insoluble magnetic and nonmagnetic metals and/or alloys [6-14]. Therefore, it does not exist only in multilayered structures. This is the reason why there has been a lot of interest. However, the transport phenomena in granular systems are not well understood. So far only a few models have been used to explain the GMR effect $[10,15,16]$. In the past studies, the magnetization of the granular films has been found to saturate at low fields, while the MR curves have shown slow saturation. Such a disagreement between MR and magnetic characteristics must first be understood if we are to understand the mechanisms responsible for the GMR effect in granular systems.

In the present study, based on experimental results for Fe-Ag films prepared under a magnetic field and $\mathrm{Co}-\mathrm{Ag} /$ permalloy double-layered films, ferromagnetic granules are shown to not be responsible for the GMR effect in granular systems. Instead, a detailed thickness dependence study indicates that the GMR effect is primarily caused by fine granules with superparamagnetic characteristics.

\section{EXPERIMENTAL}

In the double-layered structure preparation, the Co-Ag composite target ( $99.9 \%$ purity) was sputtered first using a RF method. Then, the permalloy layer, $\mathrm{Ni80Fe20}$ (wt\%, 99.9\% purity), was continuously formed onto the granular $\mathrm{Co}-\mathrm{Ag}$ layer. The preparation under a magnetic field (Hex $=0.013 \mathrm{~T}$ ) by RF sputtering was done on the Fe-Ag system. The thickness dependence was studied on the granular Fe-Ag films prepared under zero field. Si substrates used were at room temperature prior to the deposition. No attempt was made to regulate the temperature nor was the substrate temperature measured during the deposition. $A$ wavelength dispersive $x$-ray spectrometer (JEOL JXA-840) was used for elemental analyses. In this report, the composition in the samples is expressed in wt\%. Magnetization (M) and hysteresis loops were measured using a vibrating sample magnetometer (TOEI VSM-3S). The MR was measured at room temperature, about $293 \mathrm{~K}$, in a four-terminal geometry with an in-plane direct current $(\mathrm{J})$ between 0.01 and $1 \mathrm{~mA}$. A magnetic field $(\mathrm{H})$ up to $1.5 \mathrm{~T}$ was applied parallel to the current. In the present study, the MR ratio is displayed as follows:

$$
\Delta M R=[R(H)-R(1.5)] / R(1.5)
$$

where $R(1.5)$ is the $M R$ at $H=1.5 \mathrm{~T}$.

\section{RESULTS AND DISCUSSION}

As shown in Figs. 1(upper) and 1(lower), though magnetization of the granular $\mathrm{Co}-\mathrm{Ag}$ films was saturated at low fields, the MR curves showed a slow saturation. The disagreement can be emphasized by preparing a $\mathrm{Co}-\mathrm{Ag} /$ permalloy double-layered structure. The double-layered structure made the $\mathrm{M}-\mathrm{H}$. curve steep (see Fig. 1(upper)). Here, coercive force of the double-layered films, in which the thickness of the Co-Ag layer was held constant, decreased with increasing the permalloy layer thickness [17]. These 

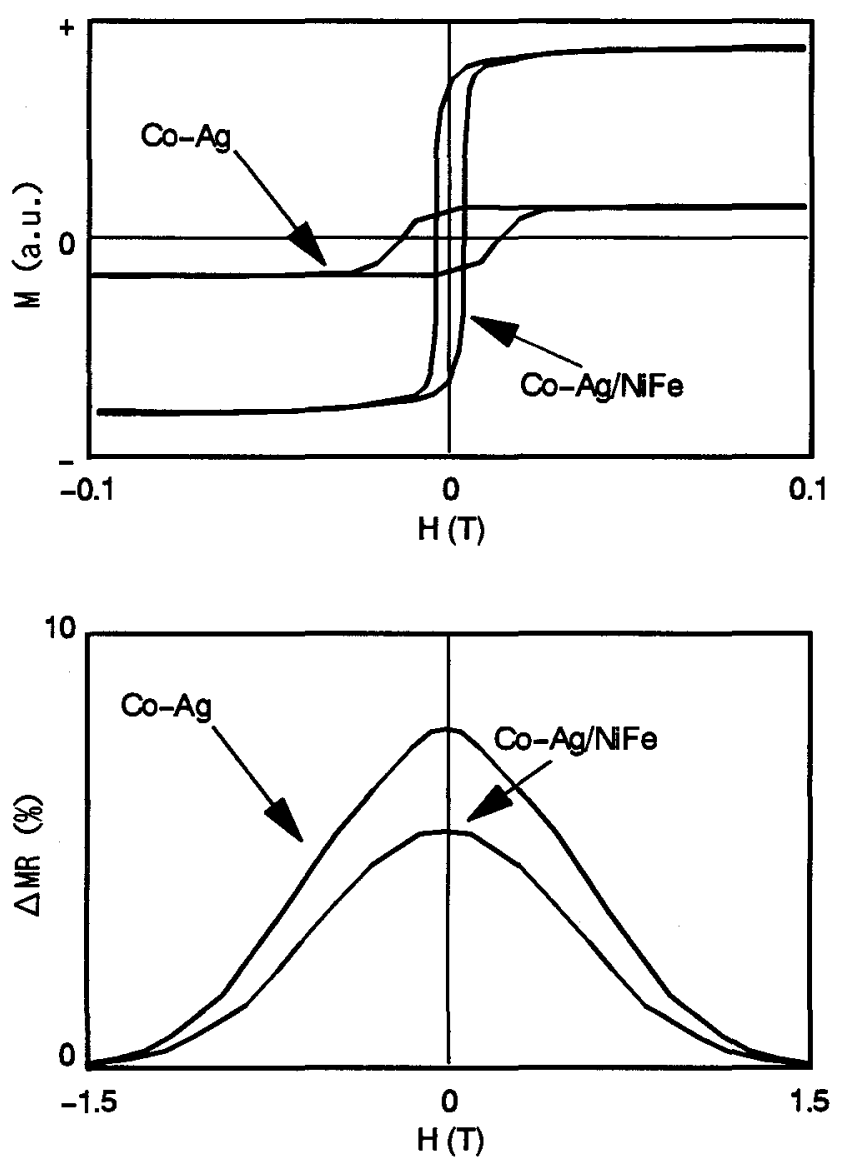

Fig. 1. M-H (upper) and MR (lower) curves at room temperature of $68 \mathrm{~nm}$-thick Co40Ag60 single-layer film and $\mathrm{Co} 40 \mathrm{Ag} 60(68 \mathrm{~nm}) / \mathrm{Ni} 80 \mathrm{Fe} 20(446 \mathrm{~nm})$ double-layered film.

indicate that the ferromagnetic granules in the $\mathrm{Co}-\mathrm{Ag}$ layer were magnetically dragged by the permalloy layer with a soft magnetic nature. To our surprise, no saturation could be observed in the MR curves of the double-layered films with a steep magnetization process, as shown in Fig. 1(lower). Specifically, after adding the permalloy layer, the shape of the MR curves changed little. In addition, MR change corresponding to the magnetization change in the magnetic field region between -0.01 and $0.01 \mathrm{~T}$ was not observed. Such disagreement between the MR and magnetic characteristics is also observed in granular $\mathrm{Fe}-\mathrm{Ag}$ films that were prepared under a magnetic field ( $\mathrm{Hex}=0.013 \mathrm{~T}$ ). Figure 2(upper) shows $\mathrm{M}-\mathrm{H}$ curves measured under the condition of $\mathrm{H}$ being applied parallel $(\mathrm{H} \| \mathrm{Hex})$ and perpendicular $(\mathrm{H} \perp \mathrm{Hex})$ to Hex. The figures indicate that magnetic anisotropy was induced in the prepared samples. In contrast, Fig. 2(lower) shows that the MR characteristics were independent of the arrangements among $\mathrm{J}, \mathrm{H}$, and Hex. This indicates that the $\mathrm{MR}$ characteristics are maintained as isotropic in samples
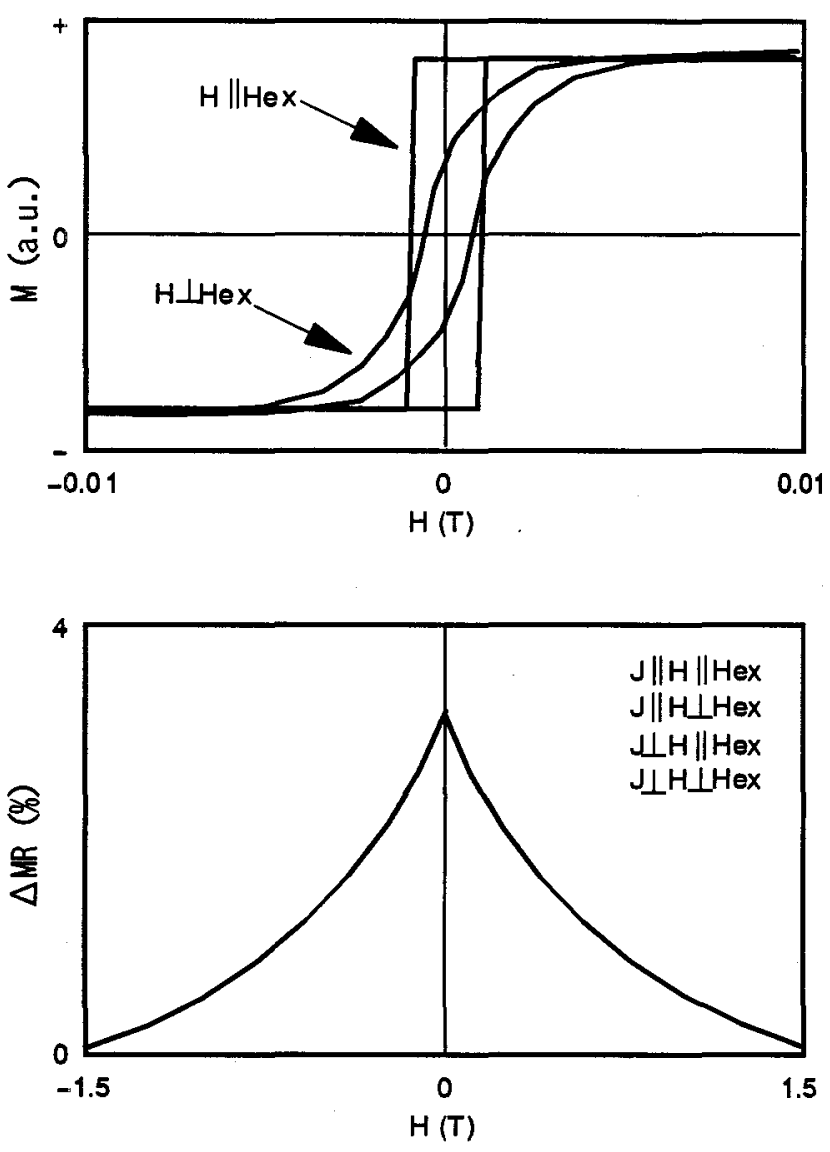

Fig. 2. M-H (upper) and MR (lower) curves at room temperature of $100 \mathrm{~nm}$-thick Fe48Ag52 film prepared under a magnetic field.

prepared under a magnetic field. Here, the MR change over the magnetic field range $-0.001-0.001 \mathrm{~T}$ was negligibly small in spite of the large magnetization change. These results show that the GMR effect is not attributable to the ferromagnetic granules. In the granular systems, therefore, there must be other magnetic components that act as scattering centers. In this respect, we note small magnetic granules with superparamagnetic characteristics. Superparamagnetic components have negligibly small contributions in the magnetic properties of granular materials, but they probably have an influence on the transport properties.

Granules have magnetic properties that change from ferromagnetic to superparamagnetic as the granule size decreases [18]. Therefore, the ratio of existing superparamagnetic granules to ferromagnetic granules is expected to increase with decreasing granular film thickness. This idea prompted us to prepare ultra-thin granular films. Figure 3 shows the thickness dependence of the MR ratio in Fe48Ag52 films prepared under zero field. The MR ratio shows a considerable increase when the thickness is less than $20 \mathrm{~nm}$. It is interesting to note 


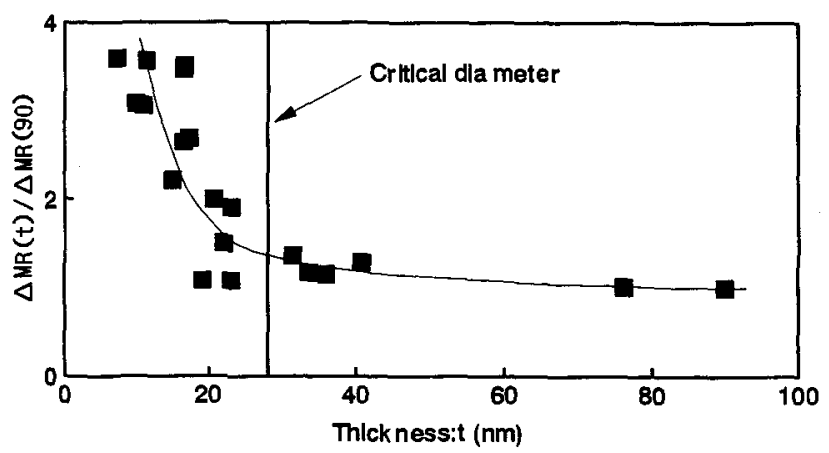

Fig. 3. MR ratios of Fe48Ag52 films with various thicknesses $(t)$. The critical diameter of $\mathrm{Fe}$ granule estimated by theoretical calculation is indicated.
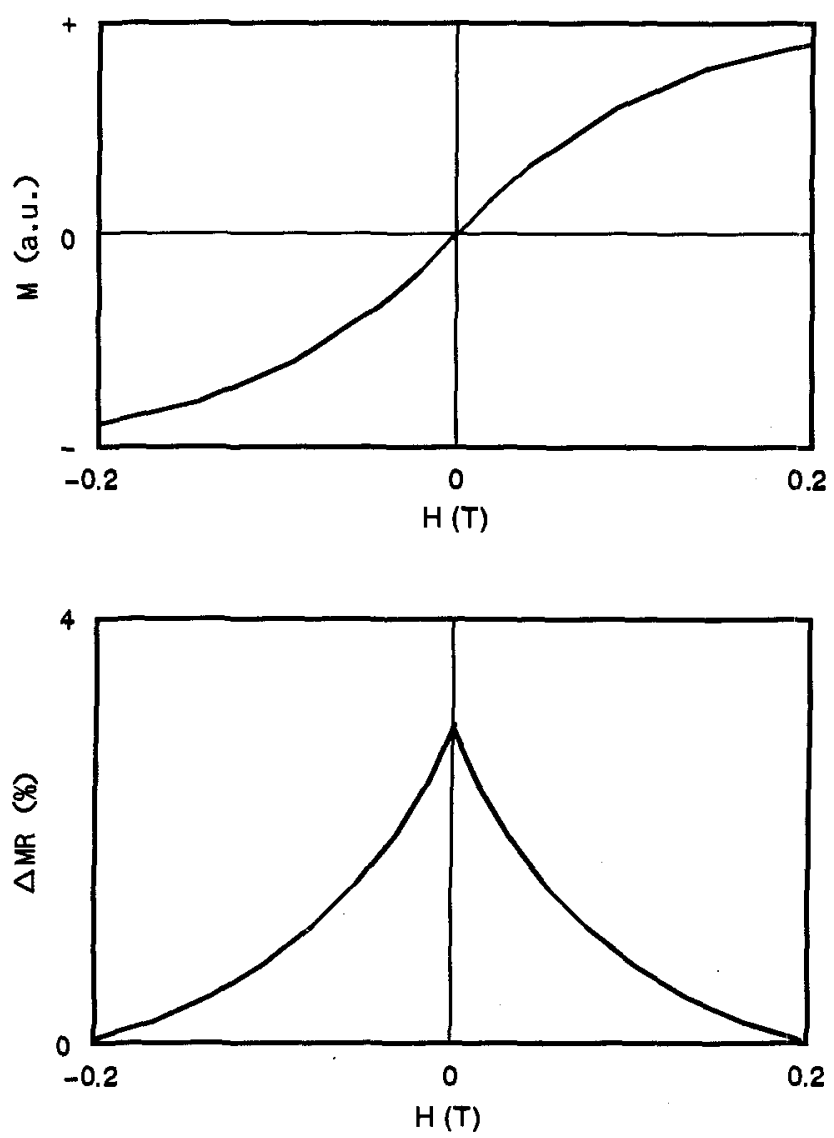

Fig. 4. $\mathrm{M}-\mathrm{H}$ (upper) and $\mathrm{MR}$ (lower) curves at room temperature of $16 \mathrm{~nm}$-thick Fe48Ag52 film.

that this thickness value of $20 \mathrm{~nm}$ is close to the critical diameter of the $\mathrm{Fe}$ granule $(25 \mathrm{~nm})$, below which the $\mathrm{Fe}$ is theoretically estimated to be superparamagnetic [19]. Here, we assert that the MR characteristics of bulk films, which are thicker than $100 \mathrm{~nm}$, were similar to those of a $90 \mathrm{~nm}$-thick film. As shown in Fig. 4, Fe48Ag52 ultra-thin films show $\mathrm{M}-\mathrm{H}$ curves with slow saturation and no hysteresis. In addition, the MR curves and $\mathrm{M}-\mathrm{H}$ curves have features that correspond well with one

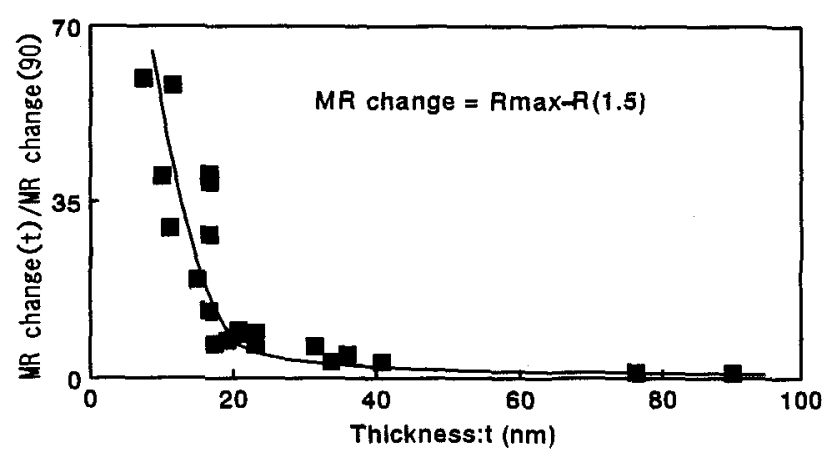

Fig. 5. MR change of Fe48Ag52 films with various thicknesses $(t)$.

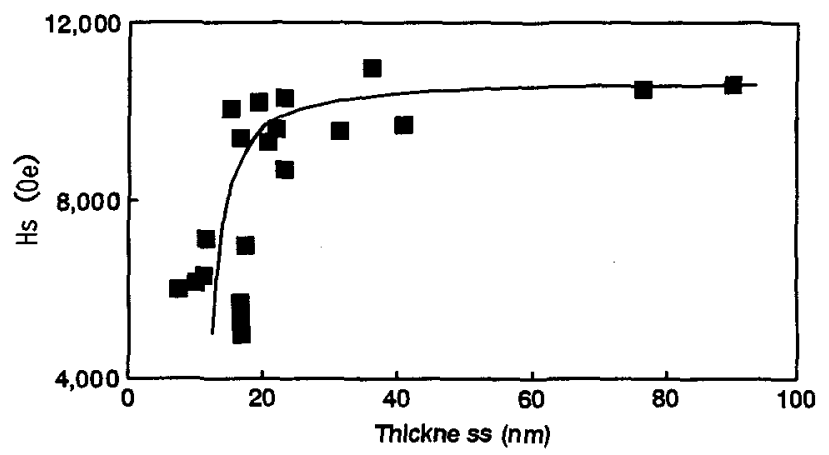

Fig. 6. Hs of Fe48Ag52 films with various thicknesses.

another. This contrasts the disagreement between MR and $\mathrm{M}-\mathrm{H}$ data in $\mathrm{Co}-\mathrm{Ag} /$ permalloy double-layered films and $\mathrm{Fe}-\mathrm{Ag}$ films prepared under a magnetic field. The above results indicate that the growth of granules is restrained with decreasing thickness as expected. They also show that small granules with superparamagnetic characteristics are the main contributors to the GMR effect in granular systems.

The value of resistance increases as required with decreasing thickness. What is surprising, however, is that the MR ratio also increases in spite of the increase in the $R(1.5)$ value. This increase indicates an extremely large intrinsic change in the $M R$ ( $R$ max $-R(1.5)$ ) for ultra-thin films. From a quantitative study, it was found that the MR change for ultra-thin films is about 60-fold that for bulk films which are thicker than $90 \mathrm{~nm}$ (see Fig. 5). In our previous study, the best result in the MR characteristics has been obtained in 100 nm-thick C019Ag81 film with a maximum MR ratio as high as 19.4\% [11]. However, the intrinsic MR change of this sample has been no more than $1 \Omega$. Since the MR change, not the MR ratio, is directly correlated with output voltage, its increase for ultra-thin films is an important knowledge for sensor device applications. Figure 6 shows the thickness dependence of the saturation field (Hs) associated with the MR 
characteristics. Here, $\mathrm{Hs}$ is defined as the value of the magnetic field corresponding to the MR ratio at a value $80 \%$ below each MR ratio (see Fig. 5 in ref.11-2). In this figure, Hs decreases with increasing MR ratio for ultra-thin films. This is probably because that the magnetic granules dispersed in the ultra-thin films construct a pseudo two-dimensional system. This idea is supported by the low Hs observed in bilayer films containing magnetic islands [20,21].

\section{CONCLUSION}

When interlayer magnetic coupling was induced in $\mathrm{Co}-\mathrm{Ag} /$ permalloy double-layered films, the change in MR was still maintained as slow. In addition, isotropic MR characteristics were observed in granular $\mathrm{Fe}-\mathrm{Ag}$ films prepared under a magnetic field. These findings show that ferromagnetic granules are not responsible for the GMR effect. The MR ratio in the Fe-Ag system was found to increase considerably when the thickness is less than $20 \mathrm{~nm}$. For such ultra-thin films, slow saturation and no hysteresis were observed in the $\mathrm{M}-\mathrm{H}$ curves, whose features correspond well with the MR curves. These results indicate that the GMR effect in granular systems is attributable to superparamagnetism. For thin-film sensor device applications, it is important that MR change increases and $\mathrm{Hs}$ decreases when the thickness of the films in granular systems decreases.

\section{REFERENCES}

[1] M. N. Baibich, J. M. Broto, A. Fert, Van Dau F. Nguyen, F. Petroff, P. Etienne, G. Creuzet, A. Friederich and J. Chazelas: Phys. Rev. Lett. 61 (1988) 2472.

[2] R. E. Camley and J. Barnas: Phys. Rev. Lett. 63 (1989) 664.

[3] P. M. Levy, S. Zhang and A. Fert: Phys. Rev. Lett. 65 (1990) 1643.
[4] J. Inoue, A. Oguri and S. Maekawa: J. Phys. Soc. Jpn. 60 (1991) 376.

[5] E. F. Fullerton, D. M. Kelly, J. Guimpell and I. K. Schuller: Phys. Rev. Lett. 68 (1992) 859.

[6] A. E. Berkowitz, J. R. Mitchell, M. J. Carey, A. P. Young, S. Zhang, F. E. Spada, F. T. Parker, A. Hutten and G. Thomas: Phys. Rev. Lett. 68 (1992) 3745.

[7] J. Q. Xiao, J. S. Jiang and C. L. Chien: Phys. Rev. Lett. 68 (1992) 3749.

[8] J. A. Barnard, A. Waknis, M. Tan, E. Haftek, M. R. Parker and M. L. Watson: J. Magn. Magn. Mater. 114 (1992) L230.

[9] J. S. Jiang, J. Q. Xiao and C. L. Chien: Appl. Phys. Lett. 61 (1992) 2362.

[10] J. Q. Wang, P. Xiong and G. Xiao: Phys. Rev. B 47 (1993) 8341.

[11] A. Maeda, M. Kume, S. Oikawa, Y. Shimizu and M. Doi: J. Phys. Condens. Matter 5 (1993) L189, 4641, 6745.

[12] M. Kitada, K. Yamamoto and N. Shimizu: J. Magn. Magn. Mater. 124 (1993) 243.

[13] N. Kataoka, H. Endo, K. Fukamichi and Y. Shimada: Jpn. J. Appl. Phys. 32 (1993) 1969.

[14] S. A. Makhlouf, K. Sumiyama, K. Wakoh, K. Suzuki, K. Takanashi and H. Fujimori: J. Magn. Magn. Mater. 126 (1993) 485.

[15] S. Zhang: Appl. Phys. Lett. 61 (1992) 1855.

[16] L. Xing and Y. C. Chang: Phys. Rev. B 48 (1993) 4156.

[17] M. Kume, A. Maeda, S. Oikawa and K. Kuroki: Jpn. J. Appl. Phys. 33 (1994) L520.

[18] W. H. Meiklejohn: Rev. Mod. Phys. 25 (1953) 302.

[19] C. Kittel: Phys. Rev, 70 (1946) 965. W. F. Brown: Phys. Rev. 105 (1957) 1479.

[20] M. Kitada: J. Magn. Magn. Mater. 123 (1993) L18.

[21] K. R. Coffey, T. L. Hylton, M. A. Parker and J. K. Howard: Appl. Phys. Lett. 63 (1993) 1579. 